\title{
DRAFT
}

\section{Path Coverings of Two Sets of Points in the Plane}

\author{
Atsushi Kaneko ${ }^{1}$, M. Kano ${ }^{2}$ and Kazuhiro Suzuki ${ }^{1}$ \\ ${ }^{1}$ Department of Computer Science and Communication Engineering \\ Kogakuin University \\ Nishi-Shinjuku, Shinjuku-ku, Tokyo 163-8677 Japan \\ e-mail: kaneko@ee.kogakuin.ac.jp \\ ${ }^{2}$ Department of Computer and Information Sciences \\ Ibaraki University, Hitachi 316-8511 Japan \\ e-mail: kano@cis.ibaraki.ac.jp
}

\begin{abstract}
We consider the following problem: For given two sets of red points and blue points in the plane respectively, we want to cover all these points with disjoint noncrossing alternating geometric paths of the same length. Determine the length of a path for which the above covering always exists under a trivial necessary condition on the numbers of red points and blue points. We give a complete solution to this problem.
\end{abstract}

\section{Introduction}

A graph drawn in the plane is called a geometric graph if every edge is a straight-line segment, and said to be non-crossing if it has no crossings. It is well-known ([8]) that for given $k$ red points and $k$ blue points in the plane in general position, there exist a non-crossing geometric alternating perfect matching on these red and blue points, that is, there exist $k$ disjoint straight-line segments that connect red points and blue points and have no crossings. Note that red and blue points are said to be in general position if no three their points lie on the same line.

We generalize the above problem by considering paths since a matching is a path of length one. A path with order $n$ and length $n-1$ is denote by $P_{n}$, and a path drawn in the plane is called an alternating path if it passes through alternately red points and blue points. We consider the following problem: For any given red and blue points in the plane in general position, do there exist disjoint non-crossing geometric alternating paths $P_{n}$ 's that cover all the red and blue points under a trivial necessary condition on the numbers 
of red points and blue points (Figure 1 (b)) ? For convenience, we briefly say that there exists a $P_{n}$-covering if there exist such disjoint paths $P_{n}$ 's. In this paper, we prove the following theorem, which gives a complete solution to the above problem.

Theorem 1 Let $g$ and $h$ denote non-negative integers. If $n$ is an even integer such that $2 \leq n \leq 14$, then for any given $(n / 2) g$ red points and $(n / 2) g$ blue points in the plane in general position, there exists a $P_{n}$-covering. If $n$ is an odd integer such that $3 \leq n \leq 11$, then for any given $\lfloor n / 2\rfloor g+\lceil n / 2\rceil h$ red points and $\lceil n / 2\rceil g+\lfloor n / 2\rfloor h$ blue points in the plane in general position, there exists a $P_{n}$-covering.

Moreover, for any integer $n$ such that $n=13$ or $n \geq 15$, there exists a configuration with $\lfloor n / 2\rfloor$ red points and $\lceil n / 2\rceil$ blue points for which there exists no $P_{n}$-covering.

In order to prove the above Theorem 1, we prove the next theorem, which is a new balanced subdivision theorem of two sets of points in the plane.

Theorem 2 Let $m \geq 1, g \geq 0$ and $h \geq 0$ be integers such that $g+h \geq 1$. Let $R$ be $a$ set of $m g+(m+1) h$ red points and $B$ a set of $(m+1) g+m h$ blue points in the plane such that no three points of $R \cup B$ lie on the same line. Then there exists a subdivision $X_{1} \cup \cdots \cup X_{g} \cup Y_{1} \cup \cdots \cup Y_{h}$ of the plane into $g+h$ disjoint convex polygons such that every $X_{i}(1 \leq i \leq g)$ contains exactly $m$ red points and $m+1$ blue points and every $Y_{j}(1 \leq j \leq h)$ contains exactly $m+1$ red points and $m$ blue points (Figure $\left.1(a)\right)$.

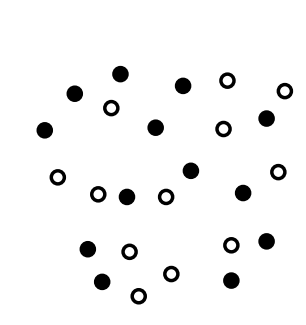

$\bullet$ :Red point $\mathbf{0}:$ Blue point

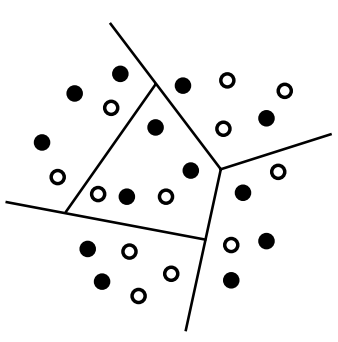

(a) A balanced subdivision

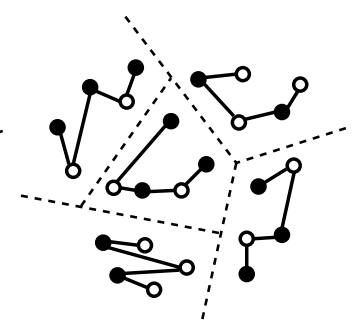

(b) A P5-covering

Figure 1: A subdivision given in Theorem 2 with $m=2, g=2, h=3$, and a $P_{5}$-covering.

It should be remarked that the above theorem 2 cannot be generalized to partitions for two positive integers $m$ and $k$ with $k \geq m+2$. Namely, if $k t+m t$ red points and $m t+k t$ blue points alternately lie on a circle in the plane for any integer $t \geq 1$, then we cannot subdivide the plane into $g+h$ disjoint convex polygons $X_{1} \cup \cdots \cup X_{g} \cup Y_{1} \cup \cdots \cup Y_{h}$ so that every $X_{i}(1 \leq i \leq g)$ contains exactly $m$ red points and $k$ blue points and every $Y_{j}(1 \leq j \leq h)$ contains exactly $k$ red points and $m$ blue points

We now explain a sketch of the proof of Theorem 1. Suppose first $n$ is even. If there exists a $P_{n}$-covering of given red and blue points in the plane, then the number of red points must be equal to that of blue points, and its number is expressed as $(n / 2) g$ for some integer $g \geq 1$. Conversely, if $(n / 2) g$ red points and $(n / 2) g$ blue points are given 
for some integer $g \geq 1$, then by Theorem 3, which will given in the next section, we can divide the plane into $g$ convex polygons so that each polygon contains exactly $n / 2$ red points and $n / 2$ blue points. Thus if we can show that for every arrangement of $n / 2$ red points and $n / 2$ blue points in the plane in general position, there exists a $P_{n}$-covering, then we can say that there exist a $P_{n}$-covering of the given red and blue points, and the problem is affirmatively solved.

Similarly, if $n$ is odd, then a trivial necessary condition for the existence of $P_{n}$-covering is that the number of red points and that of blue points are expressed as $\lfloor n / 2\rfloor g+\lceil n / 2\rceil h$ and $\lceil n / 2\rceil g+\lfloor n / 2\rfloor h$, respectively, for some non-negative integers $g$ and $h$. Conversely, if such numbers of red points and blue points are given in the plane in general position, then by Theorem 2, we can divide the plane into $g+h$ convex polygons so that each polygon contains either $\lfloor n / 2\rfloor$ red points and $\lceil n / 2\rceil$ blue points or $\lceil n / 2\rceil$ red points and $\lfloor n / 2\rfloor$ blue points. Therefore if we can show for every arrangement of $\lceil n / 2\rceil$ red points and $\lfloor n / 2\rfloor$ blue points in the plane in general position, there exists a $P_{n}$-covering, then the problem is affirmatively solved.

However, when $n=13$ or $n \geq 15$, there exist configurations of $\lceil n / 2\rceil$ red points and $\lfloor n / 2\rfloor$ blue points for which there exists no $P_{n}$-covering, and these configurations are shown in Figure 2 (a), (c) and (d).

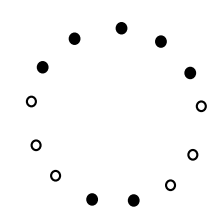

(a) $\mathrm{n}=13$

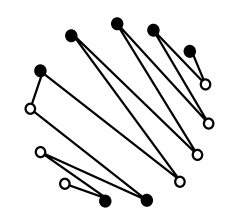

(b) $\mathrm{n}=14$

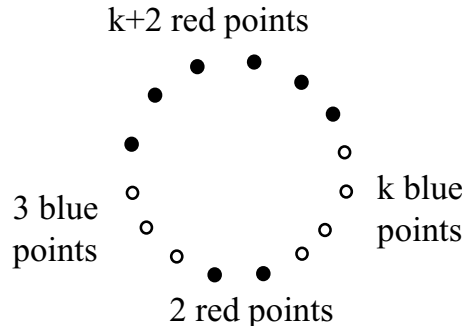

(c) $\mathrm{n}=2 \mathrm{k}+7=$ odd

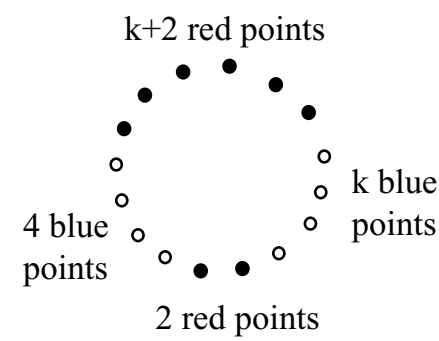

(d) $\mathrm{n}=2 \mathrm{k}+6=$ even

Figure 2: (a) A configuration of 13 points having no $P_{13}$-covering; (b) A configuration of 14 points having a $P_{14}$-covering; (c) $+(\mathrm{d})$ Configurations of 15 or more points having no $P_{n}$-covering.

\section{Proofs of Theorems}

For convenience, we call a region in the plane whose boundary consists of straight-line segments a polygon even if it is an infinite region. For example, Figure 2 (a) illustrates a subdivision of the plane into five convex polygons.

The following Theorem 3, which was conjectured in [5] and proved for $n=1,2$ in [5] and [6], was recently completely proved by Bespamyatnikh, Kirkpatrick and Snoeyink [2], Sakai [9] and by Ito, Uehara and Yokoyama [4] independently. Note that this theorem 
with $g=2$ is equivalent to the famous Ham-sandwich Theorem for the plane. Moreover, interesting results related to the next theorem can be found in [1].

Theorem 3 ([2], [9], [4]) Let $a \geq 1, b \geq 1$ and $g \geq 2$ be positive integers. Let $R$ be a set of ag red points and $B$ a set of bg blue points in the plane such that $R \cup B$ consists of points in general position. Then there exists a subdivision $X_{1} \cup X_{2} \cup \cdots \cup X_{g}$ of the plane into $g$ disjoint convex polygons such that every $X_{i}$ contains exactly a red points and $b$ blue points.

Before giving proofs, we introduce some definitions and notation. We deal only with directed lines in order to define the right side of a line and the left side of it. Thus a line means a directed line. A line $l$ dissects the plane into three pieces: $l$ and two open half-planes right $(l)$ and left $(l)$, where $\operatorname{right}(l)$ and left $(l)$ denote the open half-planes which are on the right side and the left side of $l$, respectively. Let $r_{1}$ and $r_{2}$ be two rays emanating from the same point $p$. Then we denote by $\operatorname{right}\left(r_{1}\right) \cap l e f t\left(r_{2}\right)$ the open region that is swept by the ray being rotated clockwise around $p$ from $r_{1}$ to $r_{2}$, and does not contain the point $p$ (see Figure 3). Similarly the open region left $\left(r_{1}\right) \cap \operatorname{right}\left(r_{2}\right)$ can be defined, and $r_{1} \cup r_{2}$ dissects the plane into three pieces: $r_{1} \cup r_{2}$ and two open regions $\operatorname{right}\left(r_{1}\right) \cap l e f t\left(r_{2}\right)$ and left $\left(r_{1}\right) \cap \operatorname{right}\left(r_{2}\right)$. If the internal angle $\angle r_{1} p r_{2}$ is less than $\pi$, then we call right $\left(r_{1}\right) \cap \operatorname{left}\left(r_{2}\right)$ the wedge defined by $r_{1}$ and $r_{2}$, and denote it by wedge $\left(r_{1} r_{2}\right)$ or wedge $\left(r_{2} r_{1}\right)$. For a line $l_{i}$ with suffix $i$, we define $l_{i}^{*}$ as the line lying on $l_{i}$ and having the opposite direction of $l_{i}$.
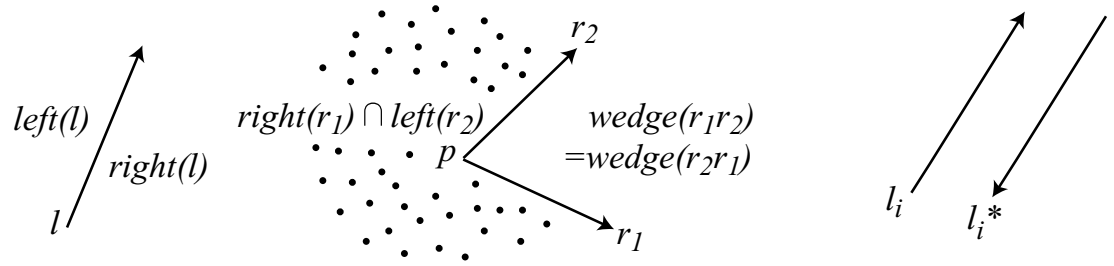

Figure 3: Open regions right $(l)$, left(l) and left( $\left.r_{1}\right) \cap \operatorname{right}\left(r_{2}\right)$, and a wedge wedge $\left(r_{1} r_{2}\right)=$ wedge $\left(r_{2} r_{1}\right)$.

Hereafter, $R$ and $B$ always denote two disjoint sets of red points and blue points in the plane, respectively, such that no three points of $R \cup B$ lie on the same line.

Theorem 4 (The Ham-sandwich Theorem [3]) For $R$ and $B$, there exists a line $l$ such that $\mid$ left $(l) \cap R|=| \operatorname{right}(l) \cap R||, l \cap R|\leq 1$,$| left (l) \cap B|=| \operatorname{right}(l) \cap B \mid$ and $|l \cap B| \leq 1$.

The line $l$ given in the above theorem is called a bisector of $R \cup B$, and we say that $R \cup B$ is bisected by $l$. It is clear that if both $|R|$ and $|B|$ are even, then the bisector $l$ passes through no red point and no blue point. The following Lemma 5 is known, and its distinct proofs are found in [5] and [2]. 
Lemma 5 For $R$ and $B$, if there exist two lines $l_{1}$ and $l_{2}$ such that $\mid$ left $\left(l_{1}\right) \cap R \mid=$ $\mid$ left $\left(l_{2}\right) \cap R \mid$ and $\mid$ left $\left(l_{1}\right) \cap B|\leq k \leq|$ left $\left(l_{2}\right) \cap B \mid$, then there exists a line $l_{3}$ such that $\mid$ left $\left(l_{3}\right) \cap R|=|$ left $\left(l_{1}\right) \cap R|$,$| left \left(l_{3}\right) \cap B \mid=k$ and $l_{3}$ passes through no point in $R \cup B$.

The following theorem, called the 3-cutting Theorem, plays an important role. This theorem was proved by Bespamyatnikh, Kirkpatrick and Snoeyink [2] under the assumption that

$$
\frac{g_{1}}{h_{1}}=\frac{g_{2}}{h_{2}}=\frac{g_{3}}{h_{3}}
$$

However this condition can be removed without changing the arguments in the proof given in [2]. This relaxation is necessary to prove our Theorem 2. Note that similar results, which seems to be essentially equivalent to the original 3-cutting Theorem, were obtained in [9] and [4], respectively.

Theorem 6 (The 3-cutting Theorem [2]) Let $g_{1}, g_{2}, g_{3}, h_{1}, h_{2}, h_{3}$ be positive integers such that $|R|=g_{1}+g_{2}+g_{3}$ and $|B|=h_{1}+h_{2}+h_{3}$. Suppose that one of the following statements (i) or (ii) is true:

(i) For every integer $i \in\{1,2,3\}$ and for every line $l$ such that $\mid$ left $(l) \cap R \mid=g_{i}$, we have $\mid$ left $(l) \cap B \mid<h_{i}$ (Figure 4 (a)).

(ii) For every integer $i \in\{1,2,3\}$ and for every line $l$ such that $\mid$ le $f t(l) \cap R \mid=g_{i}$, we have $\mid$ left $(l) \cap B \mid>h_{i}$.

Then there exists three rays emanating from a certain same point such that the three open polygon $W_{i}(1 \leq i \leq 3)$ defined by these three rays are convex, and each $W_{i}(1 \leq i \leq$ 3) contains exactly $g_{i}$ red points and $h_{i}$ blue points (Figure $\left.4(b)\right)$. Moreover, one of the three rays can be chosen to be a vertically downward ray.

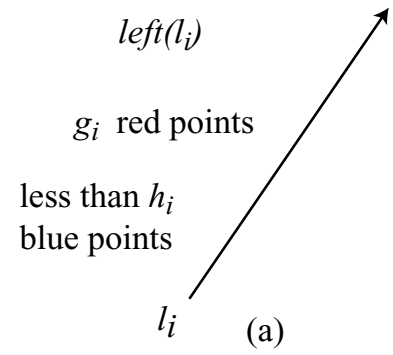

Figure 4: (a) The condition (i) ; (b) A 3-cutting.

Proof of Theorem 2. Suppose that $|R|=a g+(a+1) h$ and $|B|=(a+1) g+a h$. We prove the theorem by induction on $g+h$. In the proof, a line means a line that passes through no points in $R \cup B$, and when a line passes through some points in $R \cup B$, it is explicitly written.

If $g=0$, then $|R|=(a+1) h$ and $|B|=a h$, and so we can get the desired subdivision by Theorem 3 . Hence we may assume that $g \geq 1$, and similarly $h \geq 1$. 
Assume that there exists a line $l$ such that left $(l)$ contains exactly as $+(a+1) t$ red points and $(a+1) s+a t$ blue points for some integers $0 \leq s \leq g$ and $0 \leq t \leq h$ such that $1 \leq s+t \leq g+h-1$. Then by applying the inductive hypotheses to left $(l)$ and right $(l)$ respectively, we can obtain the desired subdivision of the plane. Hence we may assume that there exists no such a line $l$. By Lemma 5 and by this fact, for every pair $(i, j)$ of integers $0 \leq i \leq g$ and $0 \leq j \leq h$ such that $1 \leq i+j \leq g+h-1$, we can define $\operatorname{sign}(i, j)$ as follows:

$$
\begin{gathered}
\operatorname{sign}(i, j)=+ \text { if }|\operatorname{left}(l) \cap B|>(a+1) i+a j \text { for every line } l \text { with } \\
|l e f t(l) \cap R|=a i+(a+1) j \text {; and } \\
\operatorname{sign}(i, j)=- \text { if } \mid \text { left }(l) \cap B \mid<(a+1) i+a j \text { for every line } l \text { with } \\
|\operatorname{left}(l) \cap R|=a i+(a+1) j .
\end{gathered}
$$

Since $\mid$ left $(l) \cap R \mid=a s+(a+1) t$ implies $\mid$ left $\left(l^{*}\right) \cap R \mid=a(g-s)+(a+1)(h-t)$ and since $\mid$ left $(l) \cap B|+|$ left $\left(l^{*}\right) \cap B|=| B \mid$, we obtain

$$
\operatorname{sign}(g-s, h-t)=-\operatorname{sign}(s, t) \text {. }
$$

Claim 1 We may assume $\operatorname{sign}(1,0)=\operatorname{sign}(0,1)=-$.

Proof. Assume first $\operatorname{sign}(1,0)=-$. Let $l_{1}$ be a line with $\mid$ left $\left(l_{1}\right) \cap R \mid=a+1$. Let $l_{2}$ be a line which passes through one red point and satisfies the following:

$$
\left|l e f t\left(l_{2}\right) \cap R\right|=a \quad \text { and } \quad l e f t\left(l_{2}\right) \cap(R \cup B) \subseteq \operatorname{left}\left(l_{1}\right) \cap(R \cup B) \text {. }
$$

Then $\left|\operatorname{left}\left(l_{2}\right) \cap B\right|<a+1$ as $\operatorname{sign}(1,0)=-$. If $\left|\operatorname{left}\left(l_{1}\right) \cap B\right| \geq a$, then there exists a line $l_{3}$ between $l_{2}$ and $l_{3}$ such that $\left|\operatorname{left}\left(l_{3}\right) \cap R\right|=a+1$ and $\left|\operatorname{left}\left(l_{3}\right) \cap B\right|=a$, which contradicts the fact mentioned above $(s=0, t=1)$. Hence $\mid$ left $\left(l_{1}\right) \cap B \mid<a$, which implies $\operatorname{sing}(0,1)=-$.

Next assume $\operatorname{sign}(1,0)=+$. By changing the colors red and blue, we have $\operatorname{sign}(0,1)=$ -. By the same argument given above, we can show that $\operatorname{sing}(0,1)=-$ implies $\operatorname{sign}(1,0)=-$. Therefore we may assume that Claim 1 holds.

Claim 2 We may assume $\operatorname{sign}(1,0)=\cdots=\operatorname{sign}(g, 0)=-$ and $\operatorname{sing}(0,1)=\cdots=$ $\operatorname{sign}(0, h)=-$.

Proof. Suppose that there exists an integer $k(2 \leq k \leq g)$ such that $\operatorname{sign}(1,0)=\cdots=$ $\operatorname{sign}(k-1,0)=-$ and $\operatorname{sign}(k, 0)=+$. Since $\operatorname{sign}(k, 0)=+$, we have $\operatorname{sign}(g-k, h)=-$. Then

$$
\operatorname{sign}(g-k, h)=\operatorname{sign}(k-1,0)=\operatorname{sign}(1,0)=-,
$$

and thus by the 3-cutting Theorem, we can obtain a subdivision $W_{1} \cup W_{2} \cup W_{3}$ of the plane into three wedges, where $W_{1}$ contains $a(g-k)+(a+1) h$ red points and $(a+1)(g-k)+a h$ blue points, $W_{2}$ contains $a(k-1)$ red points and $(a+1) k$ blue points, and $W_{3}$ contains $a$ red points and $a+1$ blue points. By applying inductive hypotheses to each $W_{i}$, we can obtain the desired subdivision of the plane. Hence we may assume that $\operatorname{sign}(1,0)=$ $\cdots=\operatorname{sign}(g, 0)=-$, and similarly we may assume $\operatorname{sing}(0,1)=\cdots=\operatorname{sign}(0, h)=-$ by Claim 1. 
By Claim 2, we have $\operatorname{sign}(g, 0)=-$, which implies $\operatorname{sign}(0, h)=+$ by $(1)$. However, this contradicts Claim 2. Consequently Theorem 2 is proved.

Proof of Theorem 1. As we stated in the introduction, in order to prove Theorem 1, it suffices to show the next Theorem 7 .

Theorem 7 Let $n$ be an integer such that $2 \leq n \leq 12$ or $n=14$, and let $R$ be a set of $\lceil n / 2\rceil$ red points and $B$ be a set of $\lfloor n / 2\rfloor$ bule points in the plane such that no three points of $R \cup B$ lie on the same line. Then there exists a $P_{n}$-covering of $R \cup B$.

In order to prove the above Theorem 7 , we need some definitions and lemmas. For a set $X$ of points in the plane in general position, we denote by $\operatorname{conv}(X)$ the convex hull of $X$. For two points $s \notin \operatorname{conv}(X)$ and $t \in X$, we say that a vertex $t$ of conv $(X)$ is visible from $s$ if the straight-line segment st intersects $\operatorname{conv}(X)$ in exactly one point $t$, which implies that $t$ must be a vertex of $\operatorname{conv}(X)$. Let $R_{i}$ and $B_{i}$ always denote subsets of $R$ and $B$, respectively. Note the following simple lemma.

Lemma 8 Let $R$ be a set of two red points and $B$ a set of two blue points in the plane. Then for any vertex $z$ of $\operatorname{conv}(R \cup B)$, there exists a $P_{4}$-covering of $R \cup B$ starting with $z$.

The following lemma is an easy consequence of Lemma 8.

Lemma 9 Let $R$ be a set of three red points and $B$ a set of two blue points in the plane, and let $x$ be a red vertex of $\operatorname{conv}(R \cup B)$. If a blue vertex of conv $(R \cup B-\{x\})$ is visible from $x$, then there exists a $P_{5}$-covering of $R \cup B$ starting with $x$ (Figure $5(a)$ ).

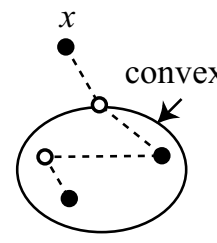

(a)

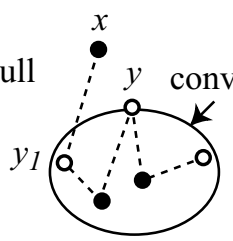

(b)

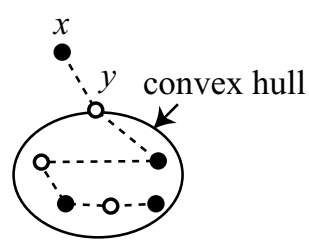

(c)

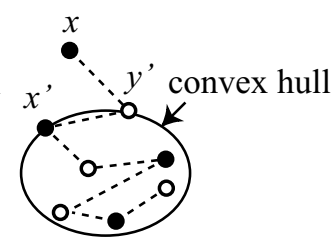

(d)

Figure 5: (a) A $P_{5}$-covering; (b) A $P_{6}$-covering; (c) A $P_{7}$-covering; (d) A $P_{8}$-covering.

Lemma 10 Let $R$ be a set of three red points and $B$ a set of three blue points in the plane, and let $x$ be a red vertex of conv $(R \cup B)$. If a blue vertex of $\operatorname{conv}(R \cup B-\{x\})$ is visible from $x$, then there exists a $P_{6}$-covering of $R \cup B$ starting with $x$ (Figure $5(b)$ ). 
Proof. Let $y$ be a blue vertex of $\operatorname{conv}(R \cup B-\{x\})$ that is visible from $x$. If a red vertex of $\operatorname{conv}(R \cup B-\{x, y\})$ is visible from $y$, then by Lemma 9 , there exists a $P_{5}$-covering of $R \cup B-\{x\}$ starting with $y$, which implies the existence of the desired $P_{6}$-covering of $R \cup B$. So we may assume that all the vertices of $\operatorname{conv}(R \cup B-\{x, y\})$ visible from $y$ are blue points. Then there are exactly two such blue vertices, and at least one of them, say $y_{1}$, is visible from $x$, and at least one red vertex of $\operatorname{conv}\left(R \cup B-\left\{x, y_{1}\right\}\right)$ is visible from $y_{1}$ (Figure $5(\mathrm{~b})$ ). Then by Lemma 9 , there exists a $P_{6}$-covering of $R \cup B$ starting with $x$.

Lemma 11 Let $R$ be a set of four red points and $B$ a set of three blue points in the plane, and let $x$ be a red vertex of $\operatorname{conv}(R \cup B)$. If a blue vertex of $\operatorname{conv}(R \cup B-\{x\})$ is visible from $x$, then there exists a $P_{7}$-covering of $R \cup B$ starting with $x$ (Figure 5 (c)).

Proof. Let $y$ be a blue vertex of $\operatorname{conv}(R \cup B-\{x\})$ that is visible from $x$. If a red vertex of $\operatorname{conv}(R \cup B-\{x, y\})$ is visible from $y$, then by applying Lemma 10 to $R \cup B-\{x\}$ and $y$, we can obtain the desired $P_{7}$-covering of $R \cup B$ starting with $x$. So we may assume that all the vertices of $\operatorname{conv}(R \cup B-\{x, y\})$ that is visible from $y$ are blue points. Then there exist exactly two such blue vertices, and at least one of them, say $y_{1}$, is visible from $x$, and at least one red vertex of $\operatorname{conv}\left(R \cup B-\left\{x, y_{1}\right\}\right)$ is visible from $y_{1}$. Then by Lemma 10, there exists a $P_{7}$-covering of $R \cup B$ starting with $x$.

Lemma 12 Let $R$ be a set of four red points and $B$ a set of four blue points in the plane, and let $x$ be a red vertex of $\operatorname{conv}(R \cup B)$. If a red vertex and a blue vertex of $\operatorname{conv}(R \cup B-\{x\})$ are both visible from $x$, then there exists a $P_{8}$-covering of $R \cup B$ starting with $x$ (Figure $5(d)$ ).

Proof. There exist a red vertex $x_{1}$ and a blue vertex $y_{1}$ of $\operatorname{conv}(R \cup B-\{x\})$ such that both of them are visible from $x$ and $x_{1} y_{1}$ is an edge of $\operatorname{conv}(R \cup B-\{x\})$. It is obvious that $x_{1}$ is a red vertex of $\operatorname{conv}\left(R \cup B-\left\{x, y_{1}\right\}\right)$ which is visible from $y_{1}$. Hence by Lemma 11, there exists the required $P_{8}$-covering of $R \cup B$ starting with $x$.

Proof of Theorem 7. Suppose that $|R|=\lceil n / 2\rceil$ and $|B|=\lfloor n / 2\rfloor$. If $2 \leq n \leq 6$, then we can easily show the existence of the required $P_{n}$-covering of $R \cup B$ by similar arguments in the case of $n=7$, which is given below. Hence we may assume that $7 \leq n \leq 12$ or $n=14$. We consider several cases corresponding to the value of $n$.

Case 1. $n=7$.

By the Ham-Sandwich Theorem, there exists a bisector $l$ such that $l$ passes through exactly one blue point, say $y$, and each of $l e f t(l)$ and right $(l)$ contains exactly two red points and one blue point. Let $R_{1} \cup B_{1}=(R \cup B) \cap \operatorname{left}(l)$. Since $y$ is a vertex of $\operatorname{conv}\left(R_{1} \cup B_{1} \cup\{y\}\right)$, by Lemma 8 there exists a $P_{4}$-covering of $R_{1} \cup B_{1} \cup\{y\}$ starting with $y$. Similarly, there exists a $P_{4}$-covering of $((R \cup B) \cap \operatorname{right}(l)) \cup\{y\}$ starting with $y$. Hence there exists the desired $P_{7}$-covering of $R \cup B$.

Case 2. $n=8$. 
Suppose that $R \cup B$ is bisected by a line $l$ so that a red vertex $x$ of $\operatorname{conv}((R \cup B) \cap l e f t(l))$ and a blue vertex $y$ of $\operatorname{conv}((R \cup B) \cap \operatorname{right}(l))$ are visible from each other (Figure 6 (a)). Then by Lemma 8 , there exist a $P_{4}$-covering of $(R \cup B) \cap l e f t(l)$ starting with $x$ and a $P_{4}$-covering of $(R \cup B) \cap \operatorname{right}(l)$ starting with $y$. By connecting these two paths by an edge $x y$, we obtain the desired $P_{8}$-covering of $R \cup B$. Hence we may assume that there exists no such a bisector $l$ of $R \cup B$.

Let $l_{1}$ be a bisector, and let $R_{1} \cup B_{1}=(R \cup B) \cap l e f t\left(l_{1}\right)$ and $R_{2} \cup B_{2}=(R \cup B) \cap$ right $\left(l_{1}\right)$. By the above assumption, if a vertex of $\operatorname{conv}\left(R_{1} \cup B_{1}\right)$ and a vertex of $\operatorname{conv}\left(R_{2} \cup B_{2}\right)$ are visible from each other, then they must have the same color. So, without loss generality, we may assume that these vertices are red. Take a tangent line to $\operatorname{conv}\left(R_{1} \cup B_{1}\right)$ and $\operatorname{conv}\left(R_{2} \cup B_{2}\right)$, which passes through two red vertices, and rotate it slightly, then we obtain a new bisector $l_{2}$ for which the partition $R \cup B=\left((R \cup B) \cap l e f t\left(l_{2}\right)\right) \cup\left((R \cup B) \cap\right.$ right $\left.\left(l_{2}\right)\right)$ does not satisfy the above assumption (Figure $6(\mathrm{~b}))$. Therefore the case is proved.

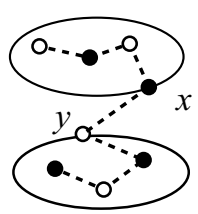

(a) $\mathrm{n}=8$

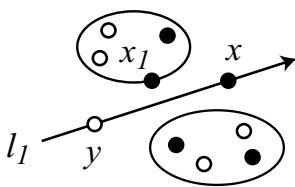

(d) $\mathrm{n}=10$

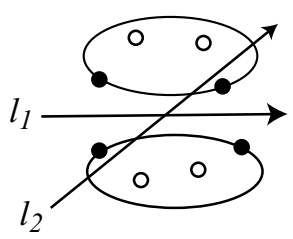

(b) $n=8$

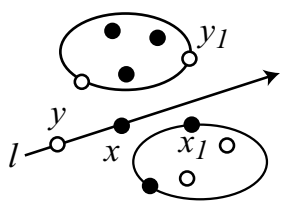

(e) $n=11$

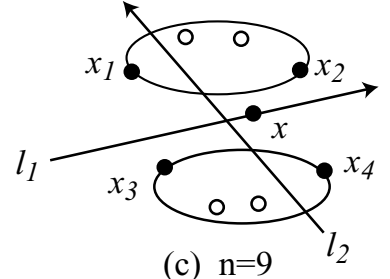

(c) $n=9$

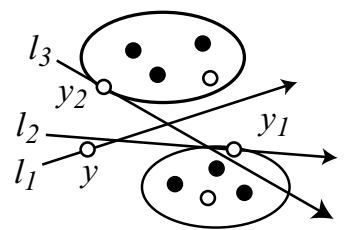

(f) $n=11$

Figure 6: (a) A $P_{8}$-covering; (b) A bisector $l_{2}$; (c) A $P_{9}$-covering; (d) A $P_{10}$-covering; (e) A $P_{11}$-covering in Subcase 5.1; (f) Bisectors $l, l_{1}, l_{2}$ in Subcase 5.2.

Case 3. $n=9$.

Let $l_{1}$ be a bisector, which passes through one red point, say $x$. Let $R_{1} \cup B_{1}=$ $(R \cup B) \cap l e f t\left(l_{1}\right)$ and $R_{2} \cup B_{2}=(R \cup B) \cap \operatorname{right}\left(l_{1}\right)$. If a blue vertex of $\operatorname{conv}\left(R_{1} \cup B_{1}\right)$ and a blue vertex of $\operatorname{conv}\left(R_{2} \cup B_{2}\right)$ are both visible from $x$, then there exists a $P_{9}$-covering of $R \cup B$ by Lemma 9. Thus we may assume that every vertex of $\operatorname{conv}\left(R_{1} \cup B_{1}\right)$ visible from $x$ is red. Hence every red point of $R_{1}=\left\{x_{1}, x_{2}\right\}$ is a vertex of $\operatorname{conv}\left(R_{1} \cup B_{1}\right)$ and visible from $x$ (Figure $6(\mathrm{c})$ ). If a blue vertex $y$ of $\operatorname{conv}\left(R_{2} \cup B_{2}\right)$ is visible from $x_{1}$ or $x_{2}$, then at least one of $y x_{1}$ and $y x_{2}$ intersects $\operatorname{conv}\left(R_{1} \cup B_{1} \cup\{x\}\right)$ in exactly one point $x_{1}$ or $x_{2}$, and so by Lemmas 8 and 10, we can obtain the desired $P_{9}$-covering of $R \cup B$. Hence we may assume that every vertex of $\operatorname{conv}\left(R_{2} \cup B_{2}\right)$ that is visible from $x_{1}$ or $x_{2}$ is red, which implies that the two red points of $R_{2}$ are vertices of $\operatorname{conv}\left(R_{2} \cup B_{2}\right)$ and visible from $x_{1}$ or 
$x_{2}$. Similarly, we may assume that every vertex of $\operatorname{conv}\left(R_{1} \cup B_{1}\right)$ visible from a red point of $R_{3}$ is red.

Let $R_{3}=\left\{x_{3}, x_{4}\right\}$. Then there exists a bisector $l_{2}$ of $R \cup B$ such that left $\left(l_{2}\right) \cap R=$ $\left\{x_{1}, x_{3}\right\}$ or $\operatorname{right}\left(l_{2}\right) \cap R=\left\{x_{2}, x_{4}\right\}$ (Figure 6 (c)). By symmetry, we may assume that $l_{2}$ satisfies left $\left(l_{2}\right) \cap R=\left\{x_{1}, x_{3}\right\}$, which implies $l_{2}$ passes through exactly one point of $\left\{x, x_{2}, x_{4}\right\}$, say $x^{\prime}$. Since a blue vertex of $\operatorname{conv}\left((R \cup B) \cap \operatorname{left}\left(l_{2}\right)\right)$ is visible from $x^{\prime}$, we can obtain a $P_{9}$-covering of $R \cup B$ by the above same argument as above.

Case 4. $n=10$.

Let $l$ be a bisector of $R \cup B$. Then $l$ passes through one red point, say $x$, and one blue point, say $y$. Let $R_{1} \cup B_{1}=(R \cup B) \cap$ left $(l)$ and $R_{2} \cup B_{2}=(R \cup B) \cap \operatorname{right}(l)$. Without loss of generality, a red vertex $x_{1}$ of $\operatorname{conv}\left(R_{1} \cup B_{1}\right)$ is visible from $y$ since otherwise a blue vertex of $\operatorname{conv}\left(R_{1} \cup B_{1}\right)$ is visible from $x$. By Lemma 9, $R_{1} \cup B_{1} \cup\{y\}$ has a $P_{5}$-covering starting with $y$ (Figure $6(\mathrm{~d})$ ). Since $x$ is a red vertex of $\operatorname{conv}\left(R_{2} \cup B_{2} \cup\{x\}\right)$ that is visible from $y$, by Lemma 10, $R_{2} \cup B_{2} \cup\{x, y\}$ has a $P_{6}$-covering starting with $y$. Consequently, $R \cup B$ has a $P_{10}$-covering.

Case $5 . \quad n=11$.

Subcase 5.1. There exists a line $l$ such that $l$ passes through one red point and one blue point, $(R \cup B) \cap l e f t(l)$ consists of three red points and two blue points, and $(R \cup B) \cap r i g h t(l)$ consists of two red points and two blue points (Figure $6(e))$.

Let $x$ and $y$ be the red point and the blue point on the line $l$, respectively, and let $R_{1} \cup B_{1}=(R \cup B) \cap$ left $(l)$ and $R_{2} \cup B_{2}=(R \cup B) \cap \operatorname{right}(l)$. If a red vertex of $\operatorname{conv}\left(R_{1} \cup B_{1}\right)$ is visible from $y$, then by Lemma $10, R_{1} \cup B_{1} \cup\{y\}$ has a $P_{6}$-covering starting with $y$. Moreover, by Lemma 10, $R_{2} \cup B_{2} \cup\{x, y\}$ has a $P_{6}$-covering starting with $y$, and so we can obtain the desired $P_{11}$-covering of $R \cup B$. Hence we may assume that every vertex of $\operatorname{conv}\left(R_{1} \cup B_{1}\right)$ visible from $y$ is blue. Similarly, if a blue vertex of $\operatorname{conv}\left(R_{2} \cup B_{2}\right)$ is visible from $x$, then by Lemma 9, $R_{2} \cup B_{2} \cup\{x\}$ has a $P_{5}$-covering starting with $x$. Moreover, by Lemma 11, $R_{1} \cup B_{1} \cup\{x, y\}$ has a $P_{7}$-covering starting with $x$, and hence there exists the desired $P_{11}$-covering of $R \cup B$. Thus we may assume that every vertex of $\operatorname{conv}\left(R_{2} \cup B_{2}\right)$ visible from $x$ is red. Therefore there exist $y_{1} \in B_{1}$ and $x_{1} \in R_{2} \cup\{x\}$ such that $y_{1} x_{1}$ intersects $\operatorname{conv}\left(R_{1} \cup B_{1} \cup\{y\}\right)$ in exactly one point $y_{1}$ and intersects $\operatorname{conv}\left(R_{2} \cup B_{2} \cup\{x\}\right)$ in exactly one point $x_{1}$ (Figure $6(\mathrm{~d})$ ). Since a red vertex of $\operatorname{conv}\left(R_{1} \cup B_{1} \cup\{y\}\right)$ is visible from $y_{1}$, by Lemma 10, $R_{1} \cup B_{1} \cup\{y\}$ has a $P_{6}$-covering starting with $y_{1}$. Similarly, $R_{2} \cup B_{2} \cup\{x\}$ has a $P_{5}$-covering starting with $x_{1}$. Therefore $R \cup B$ has the desired $P_{11}$-covering.

Subcase 5.2. There exists no line $l$ such that $l$ passes through one red point and one blue point, $(R \cup B) \cap$ left $(l)$ consists of three red points and two blue points, and $(R \cup B) \cap$ right $(l)$ consists of two red points and two blue points.

Let $l_{1}$ be a bisector, which passes through one blue point, say $y$. By the assumption of this subcase, when we rotate $l_{1}$ clockwise around $y$ until it is tangent to $\operatorname{conv}((R \cup$ $\left.B) \cap \operatorname{right}\left(l_{1}\right)\right)$ or $\operatorname{conv}\left((R \cup B) \cap \operatorname{left}\left(l_{1}\right)\right)$, it must be tangent at a blue vertex. Without 
loss of generality, we may assume that it is tangent to $\operatorname{conv}\left((R \cup B) \cap \operatorname{right}\left(l_{1}\right)\right)$ at a blue vertex, say $y_{1}$ (Figure $\left.6(\mathrm{f})\right)$. Then by a small rotation of the tangent line around $y_{1}$, we can obtain a new bisector $l_{2}$ such that $(R \cup B) \cap$ left $\left(l_{2}\right)=(R \cup B) \cap$ left $\left(l_{1}\right)$ and $(R \cup B) \cap \operatorname{right}\left(l_{2}\right)=\left((R \cup B) \cap \operatorname{right}\left(l_{1}\right)\right) \cup\{y\}-\left\{y_{1}\right\}$ (Figure $\left.6(\mathrm{f})\right)$. We repeat the above procedure one more time or two more times until we can get a bisector $l_{3}$ that passes through a blue vertex $y_{2}$ of $\operatorname{conv}\left((R \cup B) \cap \operatorname{left}\left(l_{2}\right)\right)$ (Figure $6(\mathrm{f})$ ). However, this bisector $l_{3}$ does not satisfy the assumption of this subcase, which implies that the proof of the subcase is complete.

Case 6. $n=12$.

We consider two subcases.

Subcase 6.1. There exists a line $l$ such that $l$ passes through one red point and one blue point, $(R \cup B) \cap l e f t(l)$ consists of two red points and three blue points, and $(R \cup B) \cap$ right $(l)$ consists of three red points and two blue points (Figure 7 (a)).

Let $x$ and $y$ be the red point and the blue point on $l$, respectively, and let $R_{1} \cup B_{1}=$ $(R \cup B) \cap$ left $(l)$ and $R_{2} \cup B_{2}=(R \cup B) \cap \operatorname{right}(l)$. If a blue vertex of $\operatorname{conv}\left(R_{1} \cup B_{1}\right)$ is visible from $x$, then by Lemma $10, R_{1} \cup B_{1} \cup\{x\}$ has a $P_{6}$-covering starting with $x$. Moreover, by Lemma 11, $R_{2} \cup B_{2} \cup\{x, y\}$ has a $P_{7}$-covering starting with $x$, and so there exists the desired $P_{12}$-covering of $R \cup B$. Hence we may assume that every vertex of $\operatorname{conv}\left(R_{1} \cup B_{1}\right)$ visible from $x$ is red. By symmetry, if a red vertex of $\operatorname{conv}\left(R_{2} \cup B_{2}\right)$ is visible from $y$, then we can obtain the desired $P_{12}$-covering of $R \cup B$. Hence we may assume that every vertex of $\operatorname{conv}\left(R_{2} \cup B_{2}\right)$ visible from $y$ is blue. Therefore we can find two points $x_{1} \in R_{1}$ and $y_{1} \in B_{2}$ such that $x_{1} y_{1}$ intersects $\operatorname{conv}\left(R_{1} \cup B_{1} \cup\{x\}\right)$ in exactly one point $x_{1}$ and intersects conv $\left(R_{2} \cup B_{2} \cup\{y\}\right)$ in exactly one point $y_{1}$. Since $R_{1} \cup B_{1} \cup\{x\}$ has a $P_{6}$-covering starting with $x_{1}$ and $R_{2} \cup B_{2} \cup\{y\}$ has a $P_{6}$-covering starting with $y_{1}$, we can obtain the desired $P_{12}$-covering of $R \cup B$ by connecting these paths by $x_{1} y_{1}$.

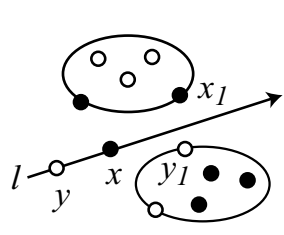

(a) $\mathrm{n}=12$

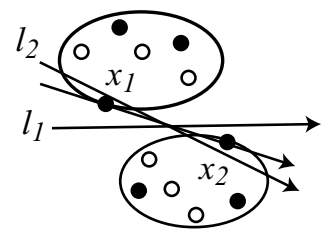

(b) $\mathrm{n}=12$

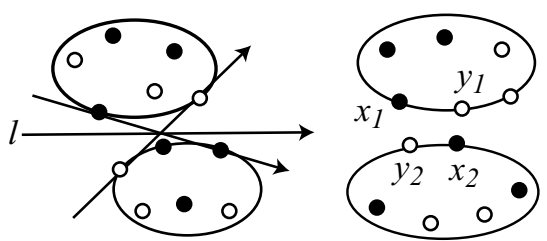

(c) $\mathrm{n}=12$

Figure 7: (a) A configuration of Subcase 6.1; (b) Bisectors $l_{1}$ and $l_{2}$; (c) A configuration of Subcase 6.2.

Subcase 6.2. There exists no line $l$ such that $l$ passes through one red point and one blue point, $(R \cup B) \cap$ left $(l)$ consists of two red points and three blue points, and $(R \cup B) \cap$ right $(l)$ consists of three red points and two blue points (Figure $7(b))$.

Let $l_{1}$ be a bisector, which passes through no red and blue points. If we rotate $l_{1}$ clockwise until it is tangent to both $\operatorname{conv}\left((R \cup B) \cap \operatorname{left}\left(l_{1}\right)\right)$ and $\operatorname{conv}((R \cup B) \cap$ 
right $\left.\left(l_{1}\right)\right)$, then the line passes through two vertices with the same colors since otherwise the assumption of the subcase does not hold for the tangent line. By a small rotation of the tangent line around its midpoint, we can obtain a new bisector $l_{2}$ (Figure 7 (b)). By repeating this procedure at most two more times, we can find a bisector $l$ such that $(R \cup B) \cap l e f t(l)$ and $(R \cup B) \cap$ right $(l)$ have two common tangent lines that pass through two red vertices or two blue vertices each (Figure 7 (c)). Let $R_{3} \cup B_{3}=(R \cup B) \cap l e f t(l)$ and $R_{4} \cup B_{4}=(R \cup B) \cap \operatorname{right}(l)$. Then we can find four points $x_{1} \in R_{3}, y_{1} \in B_{3}, x_{2} \in$ $R_{4}, y_{2} \in R_{4}$ such that $x_{1} y_{1}$ is an edge of $\operatorname{conv}\left(R_{3} \cup B_{3}\right), x_{2} y_{2}$ is an edge of $\operatorname{conv}\left(R_{4} \cup B_{4}\right)$, and $x_{1} y_{2}$ intersects $\operatorname{conv}\left(R_{3} \cup B_{3}\right)$ and $\operatorname{conv}\left(R_{4} \cup B_{4}\right)$ in exactly one point $x_{1}$ and $y_{2}$, respectively. Then by Lemma11, $R_{3} \cup B_{3}$ has a $P_{6}$-covering starting with $x_{1}$ and $R_{4} \cup B_{4}$ has a $P_{6}$-covering starting with $y_{2}$, and thus $R \cup B$ has the desired $P_{12}$-covering.

Case $7 . \quad n=14$.

Let $l$ be a bisector. Then $l$ passes through one red point, say $x$, and one blue point, say $y$. Let $R_{1} \cup B_{1}=(R \cup B) \cap l e f t(l)$ and $R_{2} \cup B_{2}=(R \cup B) \cap \operatorname{right}(l)$. By symmetry, we may assume that a red vertex $x_{1}$ of $\operatorname{conv}\left(R_{1} \cup B_{1}\right)$ is visible from both $x$ and $y$. If a blue vertex of $\operatorname{conv}\left(R_{2} \cup B_{2}\right)$ is visible from $x$, then by Lemma 11, both $R_{1} \cup B_{1} \cup\{y\}$ and $R_{2} \cup B_{2} \cup\{x\}$ have $P_{7}$-coverings starting with $y$ and $x$, respectively, and so $R \cup B$ has a $P_{14}$-covering. Hence we may assume that every vertex of $\operatorname{conv}\left(R_{2} \cup B_{2}\right)$ visible from $x$ is red, which implies that there exists a red vertex, say $x_{2}$, of $\operatorname{conv}\left(R_{2} \cup B_{2}\right)$ which is visible from both $x$ and $y$. Since $x_{2}$ is visible from $y$, by the same argument as above, we can prove that we may assume every vertex of $\operatorname{conv}\left(R_{1} \cup B_{1}\right)$ visible from $x$ is a red point. If a blue vertex of conv $\left(R_{1} \cup B_{1}\right)$ is visible from $y$, then by applying Lemma 12 to $R_{1} \cup B_{1} \cup\{y\}, R_{1} \cup B_{1} \cup\{y\}$ has a $P_{8}$-covering starting with $y$. By Lemma $11, R_{2} \cup B_{2} \cup\{y\}$ has a $P_{7}$-covering starting with $y$. Therefore $R \cup B$ has the desired $P_{14}$-covering. Thus we may assume that every vertex of $\operatorname{conv}\left(R_{1} \cup B_{1}\right)$ visible from $y$ is red. By symmetry, we may also assume that every vertex of $\operatorname{conv}\left(R_{2} \cup B_{2}\right)$ visible from $y$ is red.

We consider the two subcases.

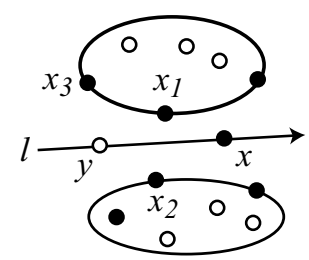

(a) $\mathrm{n}=14$

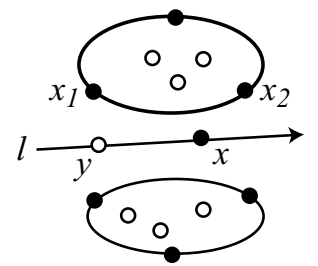

(b) $\mathrm{n}=14$

Figure 8: (a) A configuration of Subcase 7.1; (b) A configuration of Subcase 7.2; (c) A configuration of $((R \cup B) \cap l e f t(l)) \cup\{x\}$.

Subcase 7.1. Three vertices of conv $\left(R_{1} \cup B_{1}\right)$ are visible from $x$ or $y$.

Without loss of generality, we may assume that the line $l$ is horizontal and directed from left to right, and $y$ lies to the left of $x$ (Figure $7(\mathrm{a})$ ). Let $x_{3}$ be the left most vertex 
of $\operatorname{conv}\left(R_{1} \cup B_{1}\right)$ that is visible from $y$. Then $x_{3}$ is a red point, and a blue vertex of $\operatorname{conv}\left(\left(R_{1} \cup B_{1} \cup\{x\}\right)-\left\{x_{3}\right\}\right)$ is visible from $x_{3}$. Thus by Lemma 11, $R_{1} \cup B_{1} \cup\{x\}$ has a $P_{7}$-covering starting with $x_{3}$. Similarly, $R_{2} \cup B_{2} \cup\{y\}$ has a $P_{7}$-covering starting with $y$. By connecting these two paths by $x_{3} y$, we obtain the desired $P_{14}$-covering of $R \cup B$.

Subcase 7.2. Exactly two vertices of conv $\left(R_{1} \cup B_{1}\right)$ are visible from $x$ or $y$.

It is shown as in the proof of the above subcase that we may assume that the two vertices of $\operatorname{conv}\left(R_{1} \cup B_{1}\right)$ visible from $x$ or $y$ are red points (Figure $7(\mathrm{~b})$ ). Of course, these points visible from both $x$ and $y$. We denote these red points by $x_{1}$ and $x_{2}$, and the remaining red point of $R_{1}$ by $x_{3}$. By the same argument as in the proof of the above subcase, we may assume that no blue vertex of $\operatorname{conv}\left(R_{1} \cup B_{1}-\left\{x_{i}\right\}\right)$ is visible from $x_{i}$ for every $i \in\{1,2\}$ since both $x_{1}$ and $x_{2}$ are visible from $y$. However, there exists no such a configuration. Consequently the proof is complete.

\section{References}

[1] I. Bárány and J. Matoušek, Simultaneous partitions of measures by $k$-fans, Discrete Comput. Geom. 25 (2001)317-334.

[2] S. Bespamyatnikh, D. Kirkpatrick and J. Snoeyink, Generalizing ham sandwich cuts to equitable subdivisions, Discrete Comput. Geom. 24 (2000) 605-622.

[3] J. Goodman and J. O'Rourke, Handbook of Discrete and Computational Geometry, CRC Press, p.211 (1997)

[4] H. Ito, H. Uehara, and M. Yokoyama, 2-dimentinal ham-sandwich theorem for partitioning into three convex pieces, Discrete and Computational Geometry (LNCS 1763), (2000) 129-157.

[5] A. Kaneko, and M. Kano, Balanced partitions of two sets of points in the plane, Computational Geometry: Theory and Applications, 13 (1999) 253-261.

[6] A. Kaneko, and M. Kano, A balanced partition of points in the plane and tree embedding problems, preprint

[7] A. Kaneko, and M. Kano, Generalized balanced partitions of two sets of points in the plane, Discrete and Computational Geometry (LNCS 2098), (2001) 176-186.

[8] L.C. Larson, Problem-Solving Through Problems, (Springer, New York), (1983) 200201.

[9] T. Sakai, Balanced Convex Partitions of Measures in $R^{2}$, to appear in Graphs and Combinatorics. 